\title{
The Importance of being Atomic: Ecological Invasions as Random Walks instead of Waves
}

\author{
Timothy C. Reluga \\ timothy@reluga.org
}

January 28, 2016

\begin{abstract}
Invasions are one of the most easily identified spatial phenomena in ecology, and have inspired a rich variety of theories for ecologists' and naturalists' consideration. However, a number of arguments over the sensitivities of invasion rates to stochasticity, density-dependence, dimension, and discreteness persist in the literature.

The standard mathematical approach to invasions is based on Fisher's analysis of travelling waves solutions for the spread of an advantageous allele. In this paper, we exploit an alternative theory based on Ellner's premise that species invasions are best interpreted not as waves, but as random walks, and that the discreteness of living organisms is fundamentally important. Using a density-dependent invasion model in a stationary environment with indivisible (atomic) individuals where reproduction and dispersal are stochastic and independent, we show 4 key properties of Ellner's invasions previously suggested by simulation analysis: (1) greater spatial dispersal stochasticity quickens invasions, (2) greater demographic stochasticity slows invasions, (3) negative density-dependence slows invasions, and (4) greater temporal dispersal stochasticity quickens invasions. We prove the first three results by using generating functions and stochastic-dominance methods to rank furthestforward dispersal distributions. The fourth result is proven in the special case of atomless theory, but remains an open conjecture in atomic theory. In addition, we explain why, unlike atomless invasions, an infinitely wide atomic invasion in two-dimensions can travel faster than a finite-width invasion and a one-dimensional invasion. The paper concludes with a classification of invasion dynamics based on dispersal kernel tails.

Keywords: stochastic demography, invasion, long-range dispersal, wave of advance, extreme events, spreading speed
\end{abstract}

\section{Introduction}

Since ancient Greek natural philosophers Democritus and Leucippus originated the concept of an atom [Curd, 2011], there has been a tension between theories that 
treat nature as a composite of discrete particles (atoms), and theories that treat nature as a smooth continuum (atomless). During the scientific revolution, this tension took center stage in the competition between particle and wave theories of light [Inwood, 2005]. At the turn of the twentieth century, the issue reappeared as physicists speculated on the cause of the "ultraviolet catastrophe" in empirical observations of black body radiation, eventually leading to quantum theory [Kuhn, 1987]. Around the same time, Einstein and Smoluchowski were demonstrating how Fourier's continuous theory of heat propagation could be alternatively understood in terms of the Brownian motion of particles and atoms [Frey and Kroy, 2005]. And there was heated debate between Darwinians and Mendelians over whether evolution occurred through selection on distributions of continuous variation or through selection on large discrete jumps in character [Provine, 2001]. Ninety years later, in a much less famous but ecologically important discussion, Durrett and Levin [1994] emphasized how the mistaken replacement of discrete spatial structure with continuum diffusion can lead to erroneous predictions in evolutionary games. Now again, over the last two decades, controversies between continuum and discrete models have arisen in population ecologists' theories of invasions.

Invasion theory is a rich field, with a wide variety of models. Neubert and Parker [2004], Hastings et al. [2005], and Hui et al. [2011] review the general theories, while Kot et al. [2004] present a supplementary list of important theoretical results. From its birth in the 1930's until the 1990's, invasion theory was dominated by atomless models which are easy to analyze and provide elegant results [Fisher, 1937, Skellam, 1951, Barton, 1979, Okubo et al., 1989, Hosono, 1998]. In these invasion models, the population is represented as a continuous density that can be infinitely subdivided, and the advance of an invasion can often be described in terms of a travelling-wave solution. However, in the last two decades, alternative agent-based and particle models have been put forward [Ellner et al., 1998, Tang and Bennett, 2010]. In these "atomic" models, the populations are represented by sets of discrete and indivisible 
individuals. Ecologists are attracted to atomic models because of their flexibility and innate parallels with nature. Atomic models have also been studied mathematically in some contexts -- notably in reference to tribolium population dynamics [Henson et al., 2001, Scheuring and Domokos, 2005]. But overall, atomic models have received relatively less mathematical analysis, perhaps because of the different approaches they require.

The diversity of models has lead to fingering of consensus, with a variety of modelspecific results. The story begins when Mollison [1972] discovered that epidemics that dispersed according to fourth-order power-law kernels had a habit of leaping forward in large steps, in contrast to exponentially-tailed kernels where the waves of advance were more sedate. Two decades later, Kot et al. [1996] showed that heavy-tailed dispersal kernels in mean-field models could lead to invasions that progress as accelerating waves rather than waves with a constant speed. This was an exciting result with the potential to resolve Reid's paradox [Clark et al., 1998], but the result also clashed with human intuition that the spreading speed of an invasion must be bounded. Clark et al. [2001] subsequently argued that natural stochasticity stops this acceleration and only allows finite-speed invasions. Snyder [2003] performed analyses on two light-tailed dispersal kernels and found densitydependent stochastic simulations were significantly slower than integral-difference equation theory predicted [Kot, 1992] and concluded that "demographic stochasticity slows invasions". But Kot et al. [2004] countered that density-independent stochastic models show that same spreading speeds as their density-independent deterministic counterparts, while Kawasaki et al. [2006] provide an example of how stochasticity increases the spreading speed of an invasion, relative to a deterministic process with the same expectations. More recently, Brockmann and Hufnagel [2007] showed that wave fronts generated by a density-dependent particle system where particles dispersed according to Lévy flights could move with constant velocity, rather than accelerating, and Pachepsky and Levine [2011] observes that it is likely discreteness 
and density dependence that are important. Hallatschek and Fisher [2015] provide a scaling-law approach to the analysis of long-range dispersal. Jacobs and Sluckin [2015] provide an extensive simulation analysis of lattice theories, and conclude that while finite-population stochasticity slows invasions and can stop acceleration in many heavy-tailed kernels, the heaviest-tailed power-law kernels can still exhibit accelerations.

It is quite difficult to make sense of this thick body of results paper-by-paper. Different groups have adopted different models and terminologies to explain their ideas, and these differences can be challenging to reconcile. "Discrete models", for example, may refer to models that describe spread on a lattice in continuous time, spread in continuous space with difference equations, or individual-based models. "Stochasticity" may appear in confounding forms which can not be easily compared across models. And the differences between one and two dimensional models are not obvious. These obstacles have slowed our synthesis.

Yet, after digesting the menagerie of models and vague terminology, the collective mathematical research on invasions has actually lead to a relatively simple general theory. In this paper, we will lay out this proposed synthesis, using a random-walk metaphor for invasions. We will construct a nonlinear atomic stochastic process, where individuals are represented discretely. The comparison of special cases of this process shows that demographic stochasticity and negative density-dependence slow invasions, while dispersal stochasticity hastens invasions. These results are mathematically proven using stochastic ordering [Shaked, 1994], the Weierstrass's product inequality [Bromwich, 1908], and the Heyde--Schuh inequality Heyde and Schuh [1978], thus avoiding complications associated with simulations. Analysis of incrementally-shifted extreme-value statistics of different dispersal kernels under density-dependence reveals 4 separate possible regimes of behavior for an ecological invasion's random walk: (1) a regime where there is a finite upper bound on the speed of advancement, independent of density effects; (2) a regime where upper bounds 
on the advancement speed are determined by the strength of density-dependence

behind the wave-front, but progresses like a Brownian random walk; (3) a regime where upper bounds on the advancement speed are determined by the strength of density-dependence behind the wave-front, but progresses like a Lévy flight; and (4) a regime where invasions eventually accelerate past all constant upper bounds on their speed of advancement. Formal arguments supporting this classification are provided in appendices.

\section{An Atomic Spatial Model}

Let us construct a hierarchical invasion model [Jerde et al., 2009] in one spatial dimension with discrete non-overlapping generations and density-dependent mortality following settlement. The model will be atomic, in the sense that the population's state is represented by a set of points on a line rather than a continuous distribution. Each point represents the location of one individual. We will consider only the case of a homogeneous population and homogeneous space. Readers interested in heterogeneous populations should investigate matrix and integral projection extensions [Rees et al., 2014, Neubert and Caswell, 2000].

In each generation, the dispersal of offspring is described by a kernel $k$ that is independent of the parent's location, and let

$$
K(z):=\operatorname{Prob}(x<z)=\int_{-\infty}^{z} k(x) d x
$$

be the cumulative probability distribution for this dispersal kernel. The dispersal variables $y_{t, i, j}$ are the independent identically distributed (IID) dispersal distances of the $j$ th offspring of parent $i$ in generation $t\left(y_{t, i, j} \triangleleft k\right.$, which reads " $y_{t, i, j}$ is sampled from distribution $k$ ". We will adopt this uncommon notation to avoid confusion with our subsequent use of $\sim$ to represent asymptotic equivalence.).

Let the IID random variables $B_{t, i}$ be the number of offspring produced by parent $i$ 
in generation $t$. Each $B_{t, i}$ is sampled from progeny density $r\left(B_{t, i} \triangleleft r\right)$. This progeny density has probability generating function (PGF)

$$
\mathcal{R}(s):=\sum_{\ell=0}^{\infty} r_{\ell} s^{\ell}=\left\langle s^{\ell}\right\rangle \text { when } \ell \triangleleft r
$$

with $\langle\cdot\rangle$ denoting the expected value of a random variable. As with all PGF's, $\mathcal{R}(s)$ is increasing, convex and $s \in[0,1] \rightarrow \mathcal{R}(s) \in[0,1]$. The basic reproduction number $\mathscr{R}_{0}:=\mathcal{R}^{\prime}(1)$ is the expected number of progeny. Readers interested in the use of generating functions for the study of branching processes may consult any of numerous sources, including the review article by Dorman et al. [2004] and books by Matis and Kiffe [2000], Athreya and Ney [1972], Harris [1963], and Ulam [1990].

In nature, individuals often fail to reproduce, and this can lead to extinctions [Lande, 1993]. But all of our simulation experiments suggest that this complicates our invasion analysis without changing the core results. So to avoid the complications, we only consider populations where each adult produces at least one offspring:

$$
\mathcal{R}(0)=r_{0}=\operatorname{Prob}\left(B_{t, i}=0\right)=0,
$$

implying that $\mathscr{R}_{0} \geq 1$.

So far, our model has been density-independent, but we would also like to consider the sensitivity of spreading-speeds to density-dependent population regulation --progeny crowding will reduce survivorship to adulthood, and hence to speed changes. To account for this, we will adapt the concept of a "sieve", which filters out progeny who fail to meet our survival conditions, but leaves all others unchanged. This is akin to the high mortality among seedlings whose location has been determined by a dispersal process, but whose survival depends on finding a gap in a forest canopy (see Fig. 1).

Explicitly, we define a deterministic density-dependent sieve $\mathcal{F}(A)$ that, for every set of offspring locations $A$, returns the subset of locations which survive to produce 
the next generation $(\mathcal{F}(A) \subseteq A)$. The sieve will be implemented with the following algorithm: find the two closest individuals; if they are closer than a critical distance $\gamma$, remove the right-most of the two; the recalculate the distances and repeat until no two individuals are closer than $\gamma$.

The sieve $\mathcal{F}$ has a number of desirable properties. First, the sieve is the same everywhere e.g. invariant under translation, so for any displacement $d$,

$$
\mathcal{F}(A+d)=\mathcal{F}(A)+d
$$

with the additions being performed element-wise over the sets. The sieve is also local in the sense that individuals separated by a large enough gap do not change each-others survival e.g. for any $d>\gamma$ and for any two finite nonempty sets $A_{1}$ and $A_{2}$ with $\max \left(A_{1}\right)=\min \left(A_{2}\right)$,

$$
\mathcal{F}\left(A_{1} \cup\left(A_{2}+d\right)\right)=\mathcal{F}\left(A_{1}\right) \cup \mathcal{F}\left(A_{2}+d\right)
$$

The smaller the value of $\gamma$, the weaker the density-dependent mortality shadow.

It is not yet clear how to deal with more general density-dependent mortality phenomena behind an invasion front, particularly when attempting to construct lower bounds on range expansion rate. Some alternative sieves may remove dispersers expanding the range limits or even cause population extinction. Our sieve algorithm above avoids these issues and greatly simplify our mathematical analysis. The density-dependence is strictly right-sided in the sense that for every non-empty set of offspring $A$, the individual located furthest to the left (with smallest coordinate) never dies from density-dependent effects, i.e. $\min A \in \mathcal{F}(A)$. This one-sided density dependence is certainly not true in general in nature, but may be sufficiently similar to nature that the rich set of results we obtain will still hold is more realistic scenarios. The sieve algorithm also makes sure individuals are not too densely packed, i.e. for 
all offspring sets $A$ with two or more individuals over a finite space interval,

$$
|\mathcal{F}(A)| \leq \frac{(\max A-\min A)}{\gamma}+1
$$

The value $1 / \gamma$ can be interpreted as the maximum density allowed in the invasion. This density-bound acts similar to Lipschitz continuity condition employed in numerical analysis, and eliminates all situations where 2 or more individuals occupy the exact same location. The existence of a finite length scale $\gamma$ is reasonable in all real-world settings, but does rule out infinitely wide fronts invading in two or three dimensions. A non-zero density-dependence length-scale rules out planar fronts because if we sum over the extra space dimensions, we may find an infinite number of individuals in a finite interval. However, our analysis is still appropriate for any environment that is bounded in the extra dimensions, such as an environment that consists of an infinitely long strip with fixed width (see the Discussion section).

On the other hand, our sieve is unrealistic, in the sense that it is strongly leftbiased, and does not capture any density-dependent randomness in offspring survival. In what follows, we will make no explicit use of the sieve algorithm above except for simulations. Instead, we will only make use of these general properties, which are shared by a variety of algorithms. We will have to leave open the conjecture of our result's generalization to more realistic sieves.

Having dealt with the preliminaries, we now construct our model. Let $\mathscr{N}_{t}$ be the configuration of dispersed offspring from generation $t$. Without losing any generality, assume the left-most individual in the current generation is located at the origin $\left(\min \mathscr{N}_{t}=0\right)$. Offspring suffer density-dependent mortality via our sieve. Surviving offspring become reproductively mature adults. The configuration of dispersed offspring for the next generation $t+1$ (see Figure 1) is determined by the stochastic 


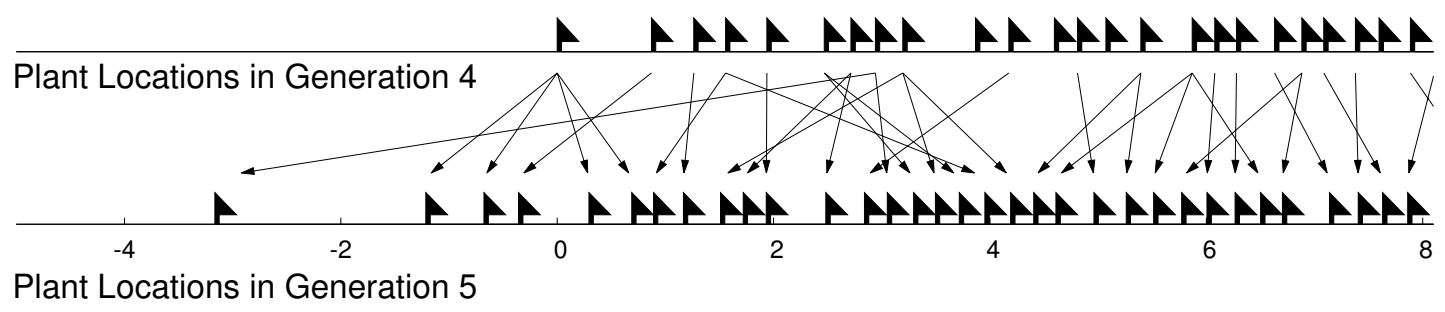

Figure 1: From the 4th to 5th generations, triangle-shaped plants invade to the left under Eq. (2.6) with Laplace-kernel $(\langle|x|\rangle=1)$ dispersal when $\mathscr{R}_{0}=4$ without demographic stochasticity. The right side of the population has been truncated, and the location scale has been shifted so the furthest forward disperser(FFD) in generation 4 is at $x=0$. The width of each lopsided plant $(\gamma=0.2)$ represents the domain where establishment is excluded by density dependence.

process

$$
\mathscr{N}_{t+1}:=\bigcup_{x_{i} \in \mathcal{F}\left(\mathscr{N}_{t}\right)}\left\{x_{i}+y_{i, j, t}: j=1 \ldots B_{t, i}\right\}, y_{i, j, t} \diamond k, B_{t, i} \diamond r
$$

or in the language of multi-type non-commuting random variables [Reluga, 2009],

$$
\mathscr{N}_{t+1}:=\mathcal{F}\left(\mathscr{N}_{t}\right) \cdot r \cdot(k+x)
$$

Within the model space of Eq. (2.6) (see Figure 2), there are several ways to arrive at distinguished limits. One way is to approximate the dispersal kernel by a comb function and to make reproduction constant so that dynamics match deterministic lattice theories where invasion occurs in fixed increments each generation. If, on the other hand, the density dependence length scale $\gamma$ is taken to zero, with everything else constant, then the population densities behind the invasion front will become large, and the dynamics may be described by spatial branching processes (which can be analyzed using deterministic probability generating functions) and nonlinear integral-difference equations. 


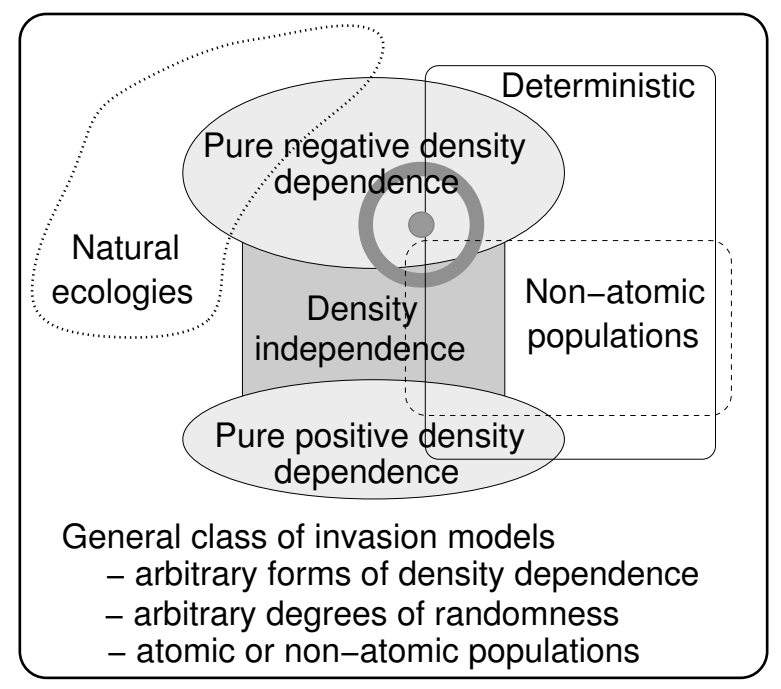

Figure 2: A Venn diagram of a topological classification of the kinds of invasion models that have been studied in stationary environments. The bullseye marks the class of models considered here, with the halo extending to asymptotic limiting cases. Atomless models form a second layer on the diagram because there exist atomless versions of each kind of model, although there are no natural ecologies that are atomless.

\section{Invasion random walks}

Having constructed our model, we now need a method for characterizing the invasion process. Invasion theory has often been approached from the perspective of travelling-wave analysis based on continuous representations of population density [Fisher, 1937, Komogorov et al., 1937, Weinberger et al., 2002]. But we may imagine an alternative metaphor [Ellner et al., 1998]: species invasion is like a random walk -- while there is a general expectation about the spreading speed of speciesoccupancy boundaries, particular invasions may randomly progress faster or slower than this expectation. Over successive generations, these random variations in spread accumulate into a random walk.

One good way to formalizing the dependence of population range on dispersal and demography has been to track the "furthest-forward disperser" (FFD) [Kot et al., 2004, Clark et al., 2001, Ellner et al., 1998, Bramson, 1978]. The position $\left(\omega_{t}\right)$ of the furthest forward individual in each generation $(t)$ defines the trajectory of species 
advancement. In each generation, the species invades with a random step size $z_{t}$ beyond its previous limits, so the range boundary $\omega_{t}$ over time $t$ progresses according to

$$
\omega_{t+1}=\omega_{t}+z_{t}
$$

We will call $z_{t}$ the invasion step. The probability distribution $p(x, t)$ of the location of the invasion front, after many steps, is approximately described by the linear advection-diffusion equation

$$
\frac{\partial p}{\partial t}+c \frac{\partial p}{\partial x}=D \frac{\partial^{2} p}{\partial x^{2}}
$$

where the diffusion rate $D:=\left[\left\langle z_{t}^{2}\right\rangle-\left\langle z_{t}\right\rangle^{2}\right] / 2$, and the advection rate $c:=\left\langle z_{t}\right\rangle$ [Weiss, 1994]. The deterministic component $c p^{\prime}$ of the invasion process convects the invasion forward at spreading speed $c$. The diffusive component $D p^{\prime \prime}$ describes the accumulation of random variation in the spread of the invasion. The randomness may lead to ranges that are ahead of or behind the expected location at any given time. In fact, this is the very pattern of invasion observed in the Tribolium experiments by Melbourne and Hastings [2009]. However, if the variance of the invasion step is infinitely large, the invasion will progress like a Lévy flight rather than a Brownian random walk. And if the expectation of the invasion step is infinitely large, the invasion process will have infinite asymptotic speed, rather than a finite speed. So, the distribution of the invasion step determines the basic properties of a single-species ecological invasion.

The key problem with this analogy is that successive invasion steps $z_{t}$ are not independent -- the invasion steps depend on the population configurations $\mathscr{N}_{t}$ which depend on the preceding generation's configurations $\mathscr{N}_{t-1}$. This interdependence is what makes the analysis of invasion processes challenging. We will have to work around this dependence by comparing spreads conditional on given population 
configurations, and by constructing bounds rather than exact results.

\subsection{Furthest-forward disperser distribution}

By deriving a distribution for the change in position $z_{t}$ of the FFD generationto-generation, we get a step-size distribution sufficient to complete our randomwalk analogy of the invasion process. Let $\mathscr{N}=\left\{x_{0}, x_{1}, \ldots x_{n}\right\}$ be a non-empty population configuration where for convenience we assume the entries are sorted

$\left(x_{0}<x_{1}<x_{2} \ldots\right)$. Our sieve ensures the inequalities are strict. Without loss of generality, we assume the location of the preceding FFD is $x_{0}=0$. We can define the invasion step $z:=\min (\mathcal{F}(\mathscr{N}) \cdot r \cdot k)$, while the cumulative probability distribution function $(\mathrm{CDF})$ of the current FFD we find $P(z \mid \mathscr{N}):=\operatorname{Prob}(\min \mathcal{F}(\mathscr{N}) \cdot r \cdot k<z)$. As always, the cumulative probability distribution is an increasing function of the invasion step $z$. Since we have assumed density-dependence is right-sided, densitydependence does not directly affect the location of the FFD. Clark et al. [2001] construct a formula for $P(z \mid \mathscr{N})$ in the case of deterministic progeny production based on knowledge of the dispersal CDF $K(x)$. A closely related formula was obtained by Pachepsky and Levine [2011]. These can be naturally extended to incorporate demographic stochasticity using the progeny PGF $\mathcal{R}(s)$; the CDF of the FFD

$$
P(z \mid \mathscr{N})=1-\prod_{x_{i} \in \mathcal{F}(\mathscr{N})} \mathcal{R}\left(1-K\left(z-x_{i}\right)\right) .
$$

As long as $\mathscr{N}$ is a finite set, we must have

$$
\lim _{z \rightarrow-\infty} P(z \mid \mathscr{N})=0
$$

And since we have assumed the sieve can not cause extinction, Eq. (2.3) implies

$$
\lim _{z \rightarrow \infty} P(z \mid \mathscr{N})=1-\mathcal{R}(0)^{|\mathscr{N}|}=1-r_{0}^{|\mathscr{N}|}=1
$$


Should anybody undertake a more complete analysis allowing for extinction, we expect our following results to remain relevant.

Throughout the subsequent conversation, we will at times refer to situations where one random variable is greater than or less than another. In these cases, the inequalities should be interpreted as an ordering in the sense of stochastic dominance [Shaked, 1994]. For example, if $x$ and $y$ are scalar random variables, then $x \prec y$ in a stochastic sense if and only if for every real number $u$, $\operatorname{Prob}(x<u)>\operatorname{Prob}(y<u)$. Note that for particular realizations of $x$ and $y$, we may have $x<y$ or $x>y$, despite having $x \prec y$.

\section{Dispersal stochasticity quickens invasions}

Stochasticity in dispersal events quickens the pace of an invasion. Why? The pace is set by the expectation of the FFD, which is an extreme event. Increasing the variation among individual dispersal events (while keeping the expectation constant) enhances the range of the extremes.

A general theorem to this affect can be proven using majorization or second-order stochasticity [Hardy et al., 1934, Rothschild and Stiglitz, 1970, Machina and Pratt, 1997, Marshall et al., 2011] (see Appendix A for details).

Proposition 1. Increases in dispersal stochasticity that do not change the expected dispersal distance increase the expected rate of invasion.

A similar effect is seen in time-varying dispersal processes [Ellner and Schreiber, $2012]$

A proof of the special case comparing the deterministic and stochastic dispersal is easier to outline. It is an elementary though less commonly stated result of statistics that (as long as all the needed expectations exist) the expectation of a minimum is 
less than the minimum of the expectations; i.e., given a set of random variables $\mathscr{N}$,

$$
\left\langle\min _{x \in \mathscr{N}} x\right\rangle \leq \min _{x \in \mathscr{N}}\langle x\rangle
$$

The invasion step is constructed as such a minimum of independent dispersal events. If dispersal is always a constant value $u$, then the invasion step must be $\langle z\rangle=u$. But if dispersal occurs with the same expectation $u$ and sufficient stochasticity, then the expectation of the invasion step must be less than the expectation of each individual dispersal event $(\langle z\rangle<u)$. Since we have adopted the convention of tracking waves moving from right to left, the stochasticity is hastening the invasion.

The effect of dispersal stochasticity is captured within density-independent branching-process theories of invasion, and consequently, within density-independent deterministic theory. Consider an invasion process with density-independent proliferation $(\mathcal{F}(A)=A)$ and a dispersal kernel that is a shifted Gauss distribution

$$
k(x)=\frac{1}{\sqrt{2 \sigma^{2} \pi}} e^{-\frac{(x-u)^{2}}{2 \sigma^{2}}} .
$$

This kernel converges to a delta-function representing deterministic dispersal as the standard deviation $\sigma$ vanishes. Using standard methods [Kot, 1992], we can show the spreading speed

$$
c^{*}=\sigma \sqrt{2 \ln \left(\mathscr{R}_{0}\right)}+u
$$

So, all else being equal, increased dispersal stochasticity quickens invasions. Clark [1998] approximates a similar result for the more general exponential power distribution.

Variation-in-space is not the only form of stochasticity present in natural dispersal processes. Variation can also occur in the timing of dispersal, with some dispersal events occurring sooner than others. Our model is poorly suited to addressing variation 
in dispersal timing because of its discrete generations, but density-dependent semiMarkov and age-dependent branching process models can account for such variation in timing [Metz et al., 2000]. It has been suggested that this form of variation also accelerates invasions (G. Jacobs, personal communications). Indeed, the kind of analysis used for Eq. (4.2) also shows that for some separable kernels, increased variation in delay accelerates invasions (see Appendix E).

Conjecture 1 (Jacobs' conjecture). Increases in the stochasticity of dispersal timing that do not change the expected dispersal time increase the expected rate of invasion.

This conjecture would, if it holds, explain the results of Kawasaki et al. [2006], whose continuous-time model comparisons emphasize stochasticity that occurs primarily in the timing of the dispersal phase.

\section{Demographic stochasticity slows invasions}

To show that demographic stochasticity slows the invasion, as Snyder [2003] argues, we compare the progression when reproduction is governed by the random variable $r$ with $\mathrm{PGF} \mathcal{R}(s)$ to the most deterministic reproduction allowed with the same expectation. Intuitively, reproduction is the most deterministic when the number of offspring is always the same e.g. if $\mathscr{R}_{0}=4$ and there are always four offspring for each parent. If the expected number of offspring is not an integer, then we sometimes have to have more or less offspring to get the averages to work out, but there is still a unique distribution that minimizes the randomness e.g. if $\mathscr{R}_{0}=3.5$, then then most deterministic offspring distribution yields 3 offspring half the time and 4 offspring half the time. In general, for a given $\mathscr{R}_{0}$, the most deterministic reproduction $\mathrm{PGF}$ is

$$
\mathcal{R}^{*}(s)=(1-\psi) s^{n-1}+\psi s^{n}
$$


where $n$ is an integer with $n-1<\mathscr{R}_{0} \leq n$ and $\psi$ is chosen so $\mathscr{R}_{0}=(1-\psi)(n-1)+\psi n$. Heyde and Schuh [1978] show that for all $s \in[0,1], \mathcal{R}^{*}(s) \leq \mathcal{R}(s)$. Then

$$
P(z \mid \mathscr{N})=1-\prod_{x_{i} \in \mathcal{F}(\mathscr{N})} \mathcal{R}\left(1-K\left(z-x_{i}\right)\right) \leq 1-\prod_{x_{i} \in \mathcal{F}(\mathscr{N})} \mathcal{R}^{*}\left(1-K\left(z-x_{i}\right)\right)
$$

Thus, the randomness in reproduction always reduces the invasion step, compared to the most deterministic possibility, and hence, slows the invasion. A more general theorem can be established again using comparative risk [Machina and Pratt, 1997] in combination with Heyde and Schuh [1978]'s result (see Appendix B).

Proposition 2. Increases in demographic stochasticity that do not change $\mathscr{R}_{0}$ slow the rate of invasion.

Intuitively, this appears to be because of the diminishing returns of extreme-value operations -- the increases in the invasion step from higher-than-expected reproduction events are outweighed by the decreases in the invasion step from lower-than-expected reproduction events.

This result seems to apply equally in density-dependent and density-independent scenarios, suggesting a contradiction with other results established for branching processes [Kot et al., 2004, Biggins, 1996]; if demographic stochasticity slows invasions, should not branching processes move more slowly than there deterministic counterparts? One possible explanation is that an invasion is not a standard random-walk, where the invasion step is independent and identically distributed each generation. Rather, the invasion step depends on the current population configuration $\mathscr{N}_{t}$ which in-turn depends on the configuration in the previous generation $\mathscr{N}_{t-1}$. In densityindependent scenarios, $z_{t}$ becomes negatively correlated over successive generations -small steps forward are offset by subsequent larger steps forward because of built-up dispersal potential in the population's configuration (see Fig. 3). An analysis conditional on a single population configuration can not capture this negative correlation. 

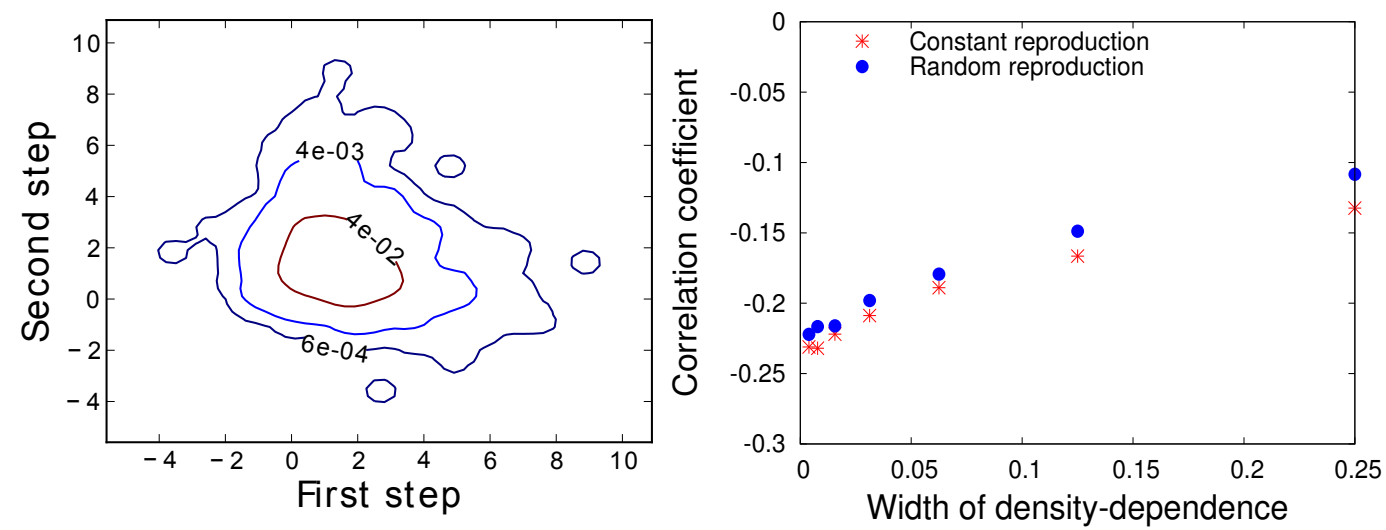

Figure 3: In an invasion, successive step sizes are not independent when densitydependence is weak -- successive steps are negatively correlated (left, kernel-smoothed scatter plot). As density-dependence strengthens ( $\gamma$ increases), the likelihood that the furthest-forward disperser comes from a parent behind the front diminishes, and the negative correlation decreases (right). Demographic randomness also appears to weaken the correlation (right). Parameters: Laplace dispersal kernel with expected dispersal distance 1 , and density-dependence width $\gamma=0.004$ (left), with 1 or 4 offspring randomly such that $\mathscr{R}_{0}=2$. Estimates based on $10^{4}$ simulation runs of 20 generations, discarding the first 10 generations as burn-in.

\section{Negative density-dependence slows an invasion}

As Snyder [2003] and Pachepsky and Levine [2011] argue, negative densitydependence slows an invasion. This is almost a trivial observation under our model. Since the density-dependent mortality imposed by our sieve leaves a subset of individuals to reproduce the next generation $\left(\mathcal{F}\left(\mathscr{N}_{t}\right) \subseteq \mathscr{N}_{t}\right)$, compared to the densityindependent situation, and since $0 \leq \mathcal{R}(1-K(z)) \leq 1$, we find

$$
P\left(z \mid \mathscr{N}_{t}\right)=1-\prod_{x_{i} \in \mathcal{F}\left(\mathscr{N}_{t}\right)} \mathcal{R}\left(1-K\left(z-x_{i}\right)\right) \leq 1-\prod_{x_{i} \in \mathscr{N}_{t}} \mathcal{R}\left(1-K\left(z-x_{i}\right)\right) .
$$

So, density-dependence always reduces the probability that the FFD disperses $z$ or more to the left compared to the density-independent case. Note that this depends intimately on the population state being atomic. Atomic density-independent populations [Kot et al., 2004] and atomless populations with negative density-dependence [Weinberger, 1982] can all invade with the same spreading speeds as density-independent 
atomless invasions. Only in atomic populations does negative density dependence slow spread.

The full importance of atomic density-dependence is best seen by considering common classes of dispersal kernels using bounding arguments in Appendix C. Generally, tail properties of dispersal kernels can be classified hierarchically based on the existence of moments and the MGF's.

Bounded kernels - The MGF is finite for all arguments $s$ and there are absolute finite limits on dispersal distance. (Ex. Top-hat distribution)

Sub-Exponential tails - The MGF is finite for all arguments $s$, but there is no strict upper bound on dispersal distance. (Ex. Gauss distribution).

Exponential tails - All moments are finite but the MGF is finite only for some arguments $s$. (Ex. Laplace distribution).

Super-exponential tails - All moments are finite but the moment generating function is only defined at $s=0($ Ex. Square-root exponentials like $c \exp (-\sqrt{|x|}))$.

Power-law tails - Most moments are infinite, and the MGF is only defined at $s=0$. (Ex. Cauchy distributions).

For simplicity, we have only considered kernels with symmetric left and right tails, but this classification can be easily generalized to handle asymmetric kernels. Collectively, bounded kernels, sub-exponentially tailed, and exponential tailed kernels are referred to as "light-tailed kernels", while kernels with super-exponential and power-law tails are referred to as "heavy-tailed kernels". Méndez et al. [2010] takes an approach to kernel tails based on a random-velocities theory using Hamilton--Jacobi equations and obtains a rather complete set of results. Here, we use an alternative approach based on elementary probability analysis from Appendix $\mathrm{C}$ to show related results summarized in Table 1. 
Table 1: Table of classification properties of invasions depending on their tail shapes. Note that this classification does not directly correspond to the kernel classes described above. Bounded diffusion refers to situations where the average behavior of the limiting form resembles standard diffusion, but absolute bounds can be placed on the maximum spread as a function of time. Boundary transitions for these asymptotic inequalities have to be handled on a case-by-case basis.

\begin{tabular}{lcc}
$\begin{array}{l}\text { Kernel tail shape } \\
\text { as } x \rightarrow-\infty\end{array}$ & Limiting Form & Maximum speed \\
\hline $0=k(x)$ & Bounded Diffusion & Density-independent \\
$0 \lesssim k(x) \lesssim A e^{-b|x|}$ & Diffusion & Density-independent \\
$A e^{-b|x|} \lesssim k(x) \lesssim A|x|^{-3}$ & Diffusion & Density-dependent \\
$A|x|^{-3} \lesssim k(x) \lesssim A|x|^{-2}$ & Lévy flight & Density-dependent \\
$A|x|^{-2} \lesssim k(x)$ & Cauchy flight & None
\end{tabular}

\subsection{Light tails}

If the dispersal kernel is bounded in the sense that dispersal never exceeds a finite distance from the source, then there will be an absolute upper bound on expected distance of the FFD $\langle z\rangle$, independent of the population distribution $\mu(x)$ describing the wave-front. So, bounded kernels always lead to finite spreading speeds. A formal justification is provided by Theorem 5 .

For sub-exponential and exponential-tailed kernels, we know from Eq. (6.1) that the density-independent special case always provides an upper bound. Based on the known results for spatial branching processes [Kot et al., 2004, Biggins, 1996], we can make use of the minimizing wave shape calculated from the deterministic speed formula [Kot, 1992, Mollison, 1972] and conclude that exponentially-tailed and sub-exponentially-tailed kernels also lead to finite-speed waves with speed-bounds that are independent of the strength of density-dependence. 


\subsection{Super-exponential tails}

Kernels with super-exponential tails have no moment-generating function, by definition. It follows from the definitions of the moment-generating function and the CDF that integrals of the form

$$
\mathscr{R}_{0} \int_{0}^{\infty} C e^{s^{*} x} K(z-x) \mathrm{d} x
$$

will diverge for all $s^{*}>0$. Thus, our upper bounds on $P(z \mid \mu)$ from Thm. 8 and Eq. (C.1) can not be applied to populations with density-independent proliferation behind the wave-front. Under density-independent proliferation, super-exponential tails lead to accelerating waves and the speed of these waves can sometimes be

characterized using the inverted dispersal kernel CDF [Kot, 1992, Mollison, 1972]. Kot et al. [1996] shows that a square-root stretched exponential kernel has a front position that progresses quadratically over time (linear acceleration).

But for an atomic population with sufficiently strong density-dependence, invasion will have a finite speed. We can show this by constructing an alternative upper bound invoking carrying-capacity limits on population size from our Lipschitz conditions on our density-dependent sieve (see Appendix C, Theorem 9).

$$
P(z \mid \mu) \lesssim \mathscr{R}_{0} K(z)+\frac{\mathscr{R}_{0}}{\gamma} \int_{0}^{\infty} K(z-x) \mathrm{d} x .
$$

As long as the kernel tails decay faster than all power-law tails, Eq. (6.3) can always be calculated and all moments for the FFD will exist, including the first and second moments of the FFD (determined by Eqs. (D.1) and (D.2), Appendix D).

\subsection{Power-law tails}

When the dispersal kernel has heavier tails, our upper bounds behave differently. Specifically, if the kernel is an asymptotic power law $\left(k(x) \sim A|x|^{-\beta}\right)$, then the 
cumulative distribution has asymptotic tail shape

$$
K(x) \sim A|x|^{1-\beta} /(\beta-1) .
$$

Our upper bound now depends on the population's configuration behind the furthest forward disperser. For the case of density-independent proliferation, invasions accelerate faster than geometrically [Kot et al., 1996]. Méndez et al. [2010] predicted and Jacobs and Sluckin [2015] observed numerically that invasions with carrying capacities exhibited finite speeds as long as $\beta>3$, but that infinite speeds appeared when $\beta<3$, even in systems with hard carrying capacities. The subsequent analysis provides further explanation.

For any exponential population distribution $(\mu(x) \propto \exp (s x))$, Eq. (6.4) implies that the integral in Eq. (C.1) diverges, so we obtain the useless bound that $P(z \mid \mu) \leq$ 1. This suggests that when proliferation is density-independent, the invasion will accelerate without bound, and analyses have born this out [Kot et al., 1996, Mancinelli et al., 2002, del Castillo-Negrete et al., 2003]. Mundt et al. [2009] claim to have observed such exponential rates of invasion in some continent-scale pathogen data sets.

Under our probabilistic model, the Weak Upper Bound Theorem (Appendix C) and Eq. (6.4) imply

$$
P(z) \lesssim \min \left\{1, \frac{\mathscr{R}_{0} A|z|^{2-\beta}}{\gamma(\beta-1)(\beta-2)}+\frac{\mathscr{R}_{0} A|z|^{1-\beta}}{\beta-1}\right\} \approx \frac{\mathscr{R}_{0} A|z|^{2-\beta}}{\gamma(\beta-1)(\beta-2)}
$$

When we construct a lower bound, we find that the $P(z)$ exhibits the same powerlaw behavior. Two important observations can be made from Eq. (6.5). Our first observation is that only a finite number of moments of the invasion step distribution are known to exist, though the number increases linearly with $\beta$. Based on Eq. (D.4), dispersal kernels with tails thinner than cubic scaling $(3<\beta)$ will be certain to possess both 1st and 2nd moments sufficient for specification of our diffusion model. 
For dispersal kernels between quadratic and cubic tail-weights $(2<\beta \leq 3)$, the first-moment exists, but the second does not, giving rise to Lévy-walk-like behavior. However, because of the natural directional asymmetry of invasion processes, these flights will appear as accelerating processes.

Our upper bound only has finite expectation when our dispersal kernels decay faster than the square of distance $(2<\beta)$. Based on our second lower bound, expressed by Theorem 5 in Appendix C, we can show that the expected value of invasion step can only be finite if for some $\zeta>0$ and some $x_{k}$,

$$
\lim _{z \rightarrow-\infty} \sum_{j=0}^{\infty} K\left(z-x_{k}-j \zeta\right)=0
$$

But for any kernel with a power law tail of $\beta \leq 2$, we have $K(z) \geq C / z$. Thus, Eq. (6.6) dominates partial sums of the harmonic series. Since Nicole Oresme showed that the summation of a harmonic series diverges [Kline, 1972], so does Eq. (6.6) diverge for all $z$. This is analytic confirmation of the observations of Jacobs and Sluckin [2015] that Cauchy kernels lead to accelerating invasions, even in the presence of density-dependence.

The second observation is that our upper bound in Eq. (6.5) depends linearly on the maximum population density $1 / \gamma$. The lower bound would also have linear dependence on carrying capacity. While we can attempt to construct sharper bounds, the presence of a lower bound with linear density-dependence is good evidence that the first moment will also have linear density-dependence on the carrying capacity behind the invasion. For heavy-tailed dispersal kernels, the expected invasion step would then increase without bound as the carrying capacity increases. Thus, a primary difference between light and heavy-tailed dispersal kernels in atomic models seems to be that for light-tailed kernels, invasion speeds can be bounded independent of carrying capacities, while for heavy-tailed kernels, invasion speeds depend on the carrying capacity. 
For the heaviest-tailed dispersal kernels, we can not construct finite moments, and expect Lévy-walk invasions no matter how strong the density dependence. If $K(z) \sim 1 / z$, then the integral in our Weak Upper Bound Theorem (Appendix C) blows up under a uniform carrying capacity assumption, and so we can not get a bound on the mean or second moment of the invasion step. This is the case when the dispersal kernel resembles a Cauchy distribution, for which the CDF is an arctangent having $1 / z$-scaling tails.

\section{Discussion}

Our analysis has established the following. Invasions with discrete generations where dispersal is independent of reproduction, negative density-dependence slows spread, as does demographic stochasticity, but dispersal stochasticity accelerates spread. For light-tailed dispersal kernels, the spreading speed will be approximately independent of the carrying capacity, but for heavy-tailed dispersal kernels, the spreading speed may depend strongly on the carrying capacity. For power-law kernels with tails heavier than $|x|^{-3}$, even strong density-dependence will not suppress the acceleration of the invasions, and all finite speeds will be eventually be exceeded.

In atomless analysis, simple invasions with concave density dependence in 2,3 , and $n$-dimensional spaces all spread asymptotically at the same speed as the 1-dimensional system. But the behavior is different in atomic invasion theory. When the spatial dimension of our atomic theory is expanded from a one-dimensional environment to a two-dimensional strip of finite width, we will still be able to derive upper and lower bounds on the invasion step, although with a correspondingly increased value for the maximum density at and behind the invasion front. However, our results will fail for an infinitely wide invasion front in two dimensions because we will not be able to construct an upper bound on the number of individuals per unit distance in the 1-dimensional profile of the invasion wave. Instead, the invasion 
step will be infinite for any dispersal kernel that does not have absolute bounds on its distance. But, for any population that is initially finite in two dimensions, deterministic density-independent models should still provide valid upper limits on the spreading speeds for exponentially bounded dispersal kernels, since two-dimensional models will still possess some negative density-dependence which can only slow the invasion process compared to density-independent spread. In two dimensions, we also often see front-roughening [Kawasaki et al., 2006], a phenomena impossible in one-dimensional theories that might be explained by the Tracy--Widom distribution and KPZ equation [O’Malley et al., 2009].

Our results have implications for some areas of network analysis. In a recent manuscript, Marvel et al. [2013] argue that the "small-world effect" commonly observed in todays interaction networks is a modern phenomena. As evidence of this, they use past studies of the Black Plague's spread across Asia and Europe in the 14th century. Based on a deterministic distributed-contacts model [Kendall, 1965, Medlock and Kot, 2003], they argue that the dispersal kernel must have been exponentially bounded to generate the constant-speed invasion observed historically, and that such a dispersal kernel could never be generated if the contact network exhibited the small-world property. While the over-all conclusion seems almost trivial, being an artifact of the dramatic increase in ease and speed of human dispersal, the argument itself is flawed. As we have shown, heavy-tailed kernels do not necessarily generate accelerating waves of spread.

If we were to extend our model to allow for the possibility that an adult fails to reproduce $\left(r_{0}>0\right)$, in all cases with a finite initial population and a densitydependent sieve meeting our conditions, the population will certainly go extinct, at which point $P(z \mid \mathscr{N})$ can no longer be defined. But we conjecture that our results should be recoverable by conditioning the invasion process on non-extinction. We must also note that our formulation using one-sided density-dependent mortality after settling is mathematically convenient, but not general; a general model would allow 
arbitrary local density-dependence. In addition, density-dependent mortality effects may appear in reproduction, dispersal, and settling phases of the life cycle. In cases where density-dependent mortality is order-preserving and monotone [Weinberger, 1982], our results should still hold, but in cases of strong or weak Allee effects, these density-dependent processes may lead to further slowing of invasion waves. In fact, many have speculated that the discreteness of individuals is analogous to the introduction of a strong Allee effect in a deterministic model. Such an analogy can not be complete, since deterministic models will not exhibit random walks, but it may still be useful.

On the other hand, one can certainly find theoretical examples, and perhaps even natural examples, where dispersal and demographic stochasticity can not be separated as we have assumed. For example, in any situation where increasing the number of dispersing propagules decreases the average dispersal distance, the effect of demographic stochasticity can not be as simple as we have modelled here. We cannot say at this time what theory modifications the coupling of reproduction and dispersal will necessitate. But the assumption of independence between demography and dispersal seems like it will be satisfactory for many applications.

Finally, our analysis fails to be fully stochastic, in that we have assumed deterministic density-dependent mortality, and not allowed for stochastic density-dependent effects. In simulations, it should be quite easy to relax this restriction, but the synthetic analysis of such processes promises to be quite rich, and while we can hope our results will continue to hold under for a wide class of such generalizations, the truth and even the accessibility of such results at the moment seems quite unknown.

\section{Acknowledgements}

I would like to thank G. Jacobs and T. Sluckin for inspiration and discussion, with additional thanks to Jan Medlock, Mark Kot, Ying Zhou, and Michael Turelli. 
Portions of this work were first presented at the University of Miami's 2014 Workshop on Mathematical Biology and Nonlinear Analysis. The research was supported in part by National Science Foundation grant CCF-1215682.

\section{References}

K. B. Athreya and P. E. Ney. Branching Processes. Springer-Verlag, New York, NY, 1972.

N. H. Barton. The dynamics of hybrid zones. Heredity, 43(3):341--359, Dec 1979. doi: 10.1038/hdy.1979.87. URL http://dx.doi.org/10.1038/hdy.1979.87.

J. D. Biggins. How fast does a general branching random walk spread? In K. B. Athreya and P. Jagers, editors, Classical and Modern Branching Processes, pages 19--40. Springer-Verlag, New York, New York, USA, 1996.

M. D. Bramson. Maximal displacement of branching Brownian motion. Communications on Pure and Applied Mathematics, 31(5):531--581, 1978 . doi: 10.1002/cpa.3160310502. URL http://dx.doi.org/10.1002/cpa.3160310502.

D. Brockmann and L. Hufnagel. Front propagation in reaction-superdiffusion dynamics: Taming levy flights with fluctuations. Physical review letters, 98(17):178301, 2007. doi: 10.1103/PhysRevLett.98.178301. URL http://dx.doi.org/10.1103/ PhysRevLett.98.178301.

T. J. Bromwich. An Introduction To The Theory Of Infinite Series. Macmillan and Company, 1908. URL https://archive.org/details/ introductiontoth00bromuoft.

J. S. Clark, M. Lewis, and L. Horvath. Invasion by extremes: population spread with variation in dispersal and reproduction. The American Naturalist, 152:204--224, 2001. URL http://www. jstor .org/stable/10.1086/319934. 
J. S. Clark. Why trees migrate so fast: Confronting theory with dispersal biology and the paleorecord. The American Naturalist, 152(2):pp. 204--224, 1998. ISSN 00030147. URL http://www.jstor.org/stable/10.1086/286162.

J. S. Clark, C. Fastie, G. Hurtt, S. T. Jackson, C. Johnson, G. A. King, M. Lewis, J. Lynch, S. Pacala, C. Prentice, et al. Reid's paradox of rapid plant migration. BioScience, 48(1):13--24, 1998. doi: 10.2307/1313224. URL http://dx.doi.org/ $10.2307 / 1313224$.

P. Curd. A Presocratics Reader (Second Edition). Hackett Publishing Co., 2 edition, 3 2011. ISBN 9781603843058. URL http://amazon.com/o/ASIN/1603843051/.

D. del Castillo-Negrete, B. Carreras, and V. Lynch. Front dynamics in reactiondiffusion systems with Lévy flights: A fractional diffusion approach. Physical Review Letters, 91(1), Jul 2003. ISSN 1079-7114. doi: 10.1103/physrevlett.91.018302. URL http://dx.doi.org/10.1103/PhysRevLett.91.018302.

K. S. Dorman, J. S. Sinsheimer, and K. Lange. In the garden of branching processes. SIAM Review, 46(2):202--229, 2004.

R. Durrett and S. Levin. The importance of being discrete (and spatial). Theoretical Population Biology, 46(3):363--394, dec 1994. doi: 10.1006/tpbi.1994.1032. URL http://dx.doi.org/10.1006/tpbi.1994.1032.

S. P. Ellner and S. J. Schreiber. Temporally variable dispersal and demography can accelerate the spread of invading species. Theoretical Population Biology, 82 (4):283--298, Dec 2012. ISSN 0040-5809. doi: 10.1016/j.tpb.2012.03.005. URL http://dx.doi.org/10.1016/j.tpb.2012.03.005.

S. P. Ellner, A. Sasaki, Y. Haraguchi, and H. Matsuda. Speed of invasion in lattice population models: pair-edge approximation. Journal of Mathematical Biology, 36(5):469--484, Mar 1998. doi: 10.1007/s002850050109. URL http: //dx.doi.org/10.1007/s002850050109. 
R. A. Fisher. The wave of advance of advantageous genes. Annals of Eugenics, 7: 353--369, 1937.

E. Frey and K. Kroy. Brownian motion: a paradigm of soft matter and biological physics. Annalen der Physik, 14(1-3):20--50, Feb 2005. doi: 10.1002/andp. 200410132. URL http://dx.doi.org/10.1002/andp. 200410132.

O. Hallatschek and D. S. Fisher. Acceleration of evolutionary spread by long-range dispersal. Proceedings of the National Academy of Sciences, 111:46 E4911-E4919, 2015. doi: 10.1073/pnas.1404663111. URL http://dx.doi.org/10.1073/pnas . 1404663111.

G. H. Hardy, J. E. Littlewood, and G. Polya. Inequalities. Cambridge University Press, 1934 .

T. E. Harris. The Theory of Branching Processes. Springer--Verlag, 1963.

A. Hastings, K. Cuddington, K. F. Davies, C. J. Dugaw, S. Elmendorf, A. Freestone, S. Harrison, M. Holland, J. Lambrinos, U. Malvadkar, B. A. Melbourne, K. Moore, C. Taylor, and D. Thomson. The spatial spread of invasions: new developments in theory and evidence. Ecology Letters, 8(1):91--101, 2005. doi: 10.1111/j.1461-0248. 2004.00687.x. URL http://dx.doi.org/10.1111/j.1461-0248.2004.00687.x.

S. M. Henson, R. F. Constantino, J. M. Cushing, R. A. Desharnais, Brian Dennis, and Aaron A. King. Lattice effects observed in chaotic dynamics of experimental populations. Science, 294:602--605, October 19 2001. doi: 10.1126/science.1063358. URL http://dx.doi.org/10.1126/science.1063358.

C. C. Heyde and H. J. Schuh. Uniform bounding of probability generating functions and the evolution of reproduction rates in birds. Journal of Applied Probability, 15 (2):243, Jun 1978. ISSN 0021-9002. doi: 10.2307/3213398. URL http://dx. doi . $\operatorname{org} / 10.2307 / 3213398$. 
Y. Hosono. The minimal speed of traveling fronts for a diffusive Lotka--Volterra competition model. Bulletin of Mathematical Biology, 60:435--448, 1998. doi: 10.1006/bulm.1997.0008. URL http://dx.doi.org/10.1006/bulm.1997.0008.

C. Hui, R. M. Krug, and D. M. Richardson. Modelling spread in invasion ecology: a synthesis. In Fifty Years of Invasion Ecology: The Legacy of Charles Elton, pages 329--43. Wiley-Blackwell, Chichester, West Sussex, 2011.

S. Inwood. The Forgotten Genius: The Biography Of Robert Hooke 1635-1703. MacAdam/Cage, 5 2005. ISBN 9781596921153.

G. S. Jacobs and T. J. Sluckin. Long-range dispersal, stochasticity and the broken accelerating wave-of-advance. Theoretical Population Biology, 100:39--55, March 2015.

C. L. Jerde, C. J. Bampfylde, and M. A. Lewis. Chance establishment for sexual, semelparous species: Overcoming the Allee effect. The American Naturalist, 173 (6):734--746, Jun 2009. doi: 10.1086/598496. URL http://dx.doi.org/10.1086/ 598496.

K. Kawasaki, F. Takasu, H. Caswell, and N. Shigesada. How does stochasticity in colonization accelerate the speed of invasion in a cellular automaton model? Ecological Research, 21(1):334--345, 2006. doi: 10.1007/s11284-006-0166-x. URL http://dx.doi.org/10.1007/s11284-006-0166-x.

D. G. Kendall. Mathematical models of the spread of infection. In Mathematics and Computer Science in Biology and Medicine, pages 213--225. H. M. Stationary Office, London, 1965.

M. Kline. Mathematical Thought from Ancient to Modern Times. Oxford Press, 1972. ISBN 0195014960. 
A. Komogorov, I. Petrovsky, and N. Piscounoff. Etude de l'equation de la diffusion aved croissance de la quantite de matiere et son application a un prbleme biologique. Moscow University, Bulletin of Mathematics, 1:1--125, 1937.

M. Kot, J. Medlock, T. Reluga, and D. B. Walton. Stochasticity, invasions, and branching random walks. Theoretical Population Biology, 66:175--184, 2004. doi: 10.1016/j.tpb.2004.05.005. URL http://dx.doi.org/10.1016/j.tpb.2004.05. 005.

M. Kot. Discrete-time travelling waves: ecological examples. Journal of Mathematical Biology, 30(4):413--436, 1992. doi: 10.1007/BF00173295. URL http://dx.doi . org/10.1007/BF00173295.

M. Kot, M. A. Lewis, and P. van den Driessche. Dispersal data and the spread of invading organisms. Ecology, 77(7):2027--2042, 1996. doi: 10.2307/2265698. URL http://dx.doi.org/10.2307/2265698.

T. S. Kuhn. Black-Body Theory and the Quantum Discontinuity, 1894-1912. University of Chicago Press, reprinted by arrangement with OUP edition, 1987. ISBN 9780226458007 .

R. Lande. Risks of population extinction from demographic and environmental stochasticity and random catastrophes. The American Naturalist, 142(6):pp. 911-927, 1993. ISSN 00030147. URL http://www.jstor.org/stable/2462690.

M. Machina and J. Pratt. Increasing risk: some direct constructions. Journal of Risk and Uncertainty, 14(2):103--127, 1997. doi: 10.1023/A:1007719626543. URL http://dx.doi.org/10.1023/A:1007719626543.

R. Mancinelli, D. Vergni, and A. Vulpiani. Superfast front propagation in reactive systems with non-gaussian diffusion. Europhysics Letters (EPL), 60(4):532--538, Nov 2002. ISSN 1286-4854. doi: 10.1209/epl/i2002-00251-7. URL http://dx. doi . org/10.1209/epl/i2002-00251-7. 
A. W. Marshall, I. Olkin, and B. C. Arnold. Inequalities: Theory of Majorization and Its Applications. Springer Series in Statistics. Springer New York, 2nd edition, 2011. ISBN http://id.crossref.org/isbn/978-0-387-68276-1. doi: 10.1007/ 978-0-387-68276-1. URL http://dx.doi .org/10.1007/978-0-387-68276-1.

S. A. Marvel, T. Martin, C. R. Doering, D. Lusseau, and M. E. J. Newman. The small-world effect is a modern phenomenon. arXiv preprint arXiv:1310.2636, 2013. URL http://arxiv.org/abs/1310.2636.

J. H. Matis and T. Kiffe. Stochastic Population Models: A Compartmental Perspective. Springer-Verlag Telos, New York, NY, 2000.

J. Medlock and M. Kot. Spreading disease: integro-differential equations old and new. Mathematical Biosciences, 184(2):201--222, 2003. doi: 10.1016/S0025-5564(03) 00041-5. URL http://dx.doi.org/10.1016/S0025-5564(03)00041-5.

B. A. Melbourne and A. Hastings. Highly variable spread rates in replicated biological invasions: Fundamental limits to predictability. Science, 325(5947):1536--1539, Sep 2009. doi: 10.1126/science.1176138. URL http://dx.doi.org/10.1126/science. 1176138.

V. Méndez, D. Campos, and I. Gómez-Portillo. Traveling fronts in systems of particles with random velocities. Physical Review E, 82(4):041119, Oct 2010. ISSN 1550-2376. doi: 10.1103/physreve.82.041119. URL http://dx.doi.org/10.1103/PhysRevE. 82.041119 .

J. A. J. Metz, D. Mollison, and F. van den Bosch. The dynamics of invasion waves. In U. Dieckmann, R. Law, and J. A. J. Metz, editors, The Geometry of Ecological Interactions, chapter 23, pages 482--512. Cambridge University Press (CUP), 2000. ISBN http://id.crossref.org/isbn/9780521022095. doi: 10.1017/cbo9780511525537. 027. URL http://dx.doi.org/10.1017/CB09780511525537.027. 
D. Mollison. The rate of spatial propagation of simple epidemics. In Proceedings of the 6th Berkeley Symposium on Mathematical Statistics and Probability, volume 3, pages 579--614. University of California Press, Berkeley, CA, 1972.

C. C. Mundt, K.E. Sackett, L.D. Wallace, C. Cowger, and J.P. Dudley. Longdistance dispersal and accelerating waves of disease: Empirical relationships. The American Naturalist, 173(4):456--466, Apr 2009. doi: 10.1086/597220. URL http://dx.doi.org/10.1086/597220.

T. K. Nayak and M. C. Christman. Effect of unequal catchability on estimates of the number of classes in a population. Scandinavian Journal of Statistics, 19(3):pp. 281--287, 1992. ISSN 03036898. URL http://www.jstor.org/stable/4616245.

M. G. Neubert and H. Caswell. Demography and dispersal: Calculation and sensitivity analysis of invasion speed for structured populations. Ecology, 81(6):1613--1628, June 2000. doi: 10.1890/0012-9658(2000)081[1613:DADCAS]2.0.CO;2. URL http: //dx.doi.org/10.1890/0012-9658(2000)081 [1613:DADCAS] 2.0.C0;2.

M. G. Neubert and I. M. Parker. Projecting rates of spread for invasive species. Risk Analysis, 24(4):817831, Aug 2004. ISSN 1539-6924. doi: 10.1111/j.0272-4332.2004. 00481.x. URL http://dx.doi.org/10.1111/j.0272-4332.2004.00481.x.

A. Okubo, P. K. Maini, M. H. Williamson, and J. D. Murray. On the spatial spread of the gray squirrel in Britain. Proceedings of the Royal Society of London, B238: 113--125, 1989. doi: 10.1098/rspb.1989.0070. URL http://dx.doi.org/10.1098/ rspb.1989.0070.

L. O’Malley, G Korniss, and T. Caraco. Ecological invasion, roughened fronts, and a competitors extreme advance: Integrating stochastic spatial-growth models. Bulletin of Mathematical Biology, 71:1160--1188, 2009. doi: 10.1007/s11538-009-9398-6. URL http://dx.doi.org/10.1007/s11538-009-9398-6. 
E. Pachepsky and J. M Levine. Density dependence slows invader spread in fragmented landscapes. The American Naturalist, 177(1):18--28, 2011. doi: 10.1086/657438. URL http://dx.doi.org/10.1086/657438.

W. B. Provine. The Origins of Theoretical Population Genetics. University of Chicago Press, 1 edition, 1 2001. ISBN 9780226684642. URL http://amazon.com/o/ASIN/ $0226684644 /$.

M. Rees, D. Z. Childs, and S. P. Ellner. Building integral projection models: a users guide. Journal of Animal Ecology, 83(3):528--545, Jan 2014. ISSN 0021-8790. doi: 10.1111/1365-2656.12178. URL http://dx.doi .org/10.1111/1365-2656.12178.

T. C. Reluga. Branching processes and non-commuting random variables in population biology. Canadian Applied Math Quarterly, 17(2):387, 2009. URL http://www . math.ualberta.ca/ami/CAMQ/table_of_content/vol_17_2.html.

M. Rothschild and J. E. Stiglitz. Increasing risk: I. a definition. Journal of Economic Theory, 2(3):225--243, 1970. doi: 10.1016/0022-0531(70)90038-4. URL http: //dx.doi.org/10.1016/0022-0531(70)90038-4.

I. Scheuring and G. Domokos. Sturdy cycles in the chaotic tribolium castaneum data series. Theoretical Population Biology, 67(2):127--139, Mar 2005. doi: 10.1016/j. tpb.2004.11.002. URL http://dx.doi.org/10.1016/j.tpb.2004.11.002.

M. Shaked. Stochastic Orders and Their Applications (Probability and Mathematical Statistics). Academic Press, 6 1994. ISBN 9780126381603.

J. G. Skellam. Random dispersal in theoretical populations. Biometrika, 38:196-218, 1951. doi: 10.1093/biomet/38.1-2.196. URL http://dx.doi.org/10.1093/ biomet/38.1-2.196.

R. E. Snyder. How demographic stochasticity can slow biological invasions. Ecology, 84:1333--1339, 2003. doi: 10.1890/0012-9658(2003)084[1333:HDSCSB]2.0.CO; 
2. URL http://dx.doi.org/10.1890/0012-9658(2003)084[1333:HDSCSB] 2.0. $\mathrm{CO} ; 2$.

W. Tang and D. A. Bennett. Agent-based modeling of animal movement: A review. Geography Compass, 4(7):682--700, Jul 2010. ISSN 1749-8198. doi: 10.1111/j. 1749-8198.2010.00337.x. URL http://dx.doi.org/10.1111/j.1749-8198.2010. $00337 . \mathrm{x}$

Y. L. Tong. Some majorization orderings of heterogeneity in a class of epidemics. J. Appl. Probab., 34(1):84--93, 1997. ISSN 0021-9002. URL http://www.jstor.org. ezaccess.libraries.psu.edu/stable/3215177.

S. M. Ulam. Analogies Between Analogies: The mathematical reports of S. M. Ulam and his Los Alamos collaborators. Los Alamos Series in Basic and Applied Sciences. University of California Press, 1990.

H. F. Weinberger. Long-time behavior of a class of biological models. SIAM Journal on Mathematical Analysis, 13(3):353--396, May 1982. doi: 10.1137/0513028. URL http://epubs.siam.org/doi/abs/10.1137/0513028.

H. F. Weinberger, M. A. Lewis, and B. Li. Analysis of the linear conjecture for spread in cooperative models. Journal of Mathematical Biology, 45:183--218, 2002. doi: 10.1007/s002850200145. URL http://dx.doi.org/10.1007/s002850200145.

G. H. Weiss. A primer of random walkology. In A. Bunde and S. Havlin, editors, Fractals in Science, pages 119--162. Springer Berlin Heidelberg, 1994. ISBN http://id.crossref.org/isbn/978-3-642-77953-4. doi: 10.1007/978-3-642-77953-4_5. URL http://dx.doi.org/10.1007/978-3-642-77953-4_5. 


\section{A Dispersal stochasticity}

In order to make Proposition 1 precise, we must define "greater variation in dispersal". One very powerful way to do this is with "comparative risk" [Machina and Pratt, 1997], which is closely related to second-order stochastic dominance Rothschild and Stiglitz [1970], as well as majorization [Hardy et al., 1934, Marshall et al., 2011]. Comparative risk was designed to recognize situations where probability densities have the same expectation, but one has greater variation in the tails than another (hence generating greater variation around the mean). It has been previously considered in population biology [Nayak and Christman, 1992, Tong, 1997], though does not seem to have been employed in invasion theory yet.

Suppose we are given two dispersal kernels $k(x)$ and $\ell(x)$ with the same expected value. From Eq. (D.1), equality in expected value implies

$$
f_{-\infty}^{\infty} \int_{-\infty}^{v} k(u)-\ell(u) \mathrm{d} u \mathrm{~d} v=0
$$

Now, we say $\ell$ majorizes $k(\ell \succcurlyeq k)$ if and only if for every real number $x$,

$$
\int_{-\infty}^{x} \int_{-\infty}^{v} \ell(u) \mathrm{d} u \mathrm{~d} v \geq \int_{-\infty}^{x} \int_{-\infty}^{v} k(u) \mathrm{d} u \mathrm{~d} v
$$

with strict inequality holding for some finite value of $x$. This appears at first to be an odd definition, but as Rothschild and Stiglitz [1970] show, Equation (A.2) is equivalent to saying that the dispersal kernel $\ell$ majorizes $k$ if and only if $\ell$ can be created from $k$ by a secondary dispersal process with no bias i.e. $\ell \sim k+\epsilon$ in random-variable notation, where $\epsilon$ can depend on the value of $k$, but $E(\epsilon)=0$ for all $k$ ). So $\ell \succcurlyeq k$ when $\ell$ has greater variation, but the same expectation.

We can now establish that greater dispersal variation in the sense of Eq. (A.2) leads to greater invasion speeds. Our result probably appears in [Marshall et al., 2011], and elsewhere, but we present our own argument for completeness. First, we 
establish as a widely known lemma that conditional expectation preserves concavity.

Lemma 1. If $f(x, y)$ is concave, then $E(f(x, y) \mid x)$ is concave in $x$.

Proof. Given a concave function $f(x, y)$ and $p \geq 0, q \geq 0$ such that $p+q=1$, then, by definition, for any $x_{p}, x_{q}$,

$$
p f\left(x_{p}, y\right)+q f\left(x_{q}, y\right) \leq f\left(p x_{p}+q x_{q}, y\right) .
$$

Integrating both sides over a given conditional probability distribution $p(y \mid x)$,

$$
\int\left[p f\left(x_{p}, y\right)+q f\left(x_{q}, y\right)\right] p(y \mid x) \mathrm{d} y \leq \int f\left(p x_{p}+q x_{q}, y\right) p(y \mid x) \mathrm{d} y
$$

Using the definition of conditional expectation $E(f(x, y) \mid x)=\int f(x, y) p(y \mid x) \mathrm{d} y$, it follows that

$$
p E\left(f(x, y) \mid x=x_{p}\right)+q E\left(f(x, y) \mid x=x_{q}\right) \leq E\left(f(x, y) \mid x=p x_{p}+q x_{q}\right)
$$

Thus, by definition, $E(f(x, y) \mid x)$ must be concave in $x$.

We now establish our main theorem.

Theorem 2. If $\ell$ and $k$ represent dispersal distributions where $\ell$ majorizes $k$ ( $\ell \succcurlyeq k$ ) then $\forall x \in \mathbb{R}^{n}$,

$$
E\left(\min \left\{\ell_{0}+x_{0}, . ., \ell_{n}+x_{n}\right\} \mid x\right)<E\left(\min \left\{k_{0}+x_{0}, . ., k_{n}+x_{n}\right\} \mid x\right)
$$

Proof. Let $F_{i}:=E\left(\min \left\{\ell_{0}+x_{0}, \ell_{1}+x_{1}, \ldots \ell_{i-1}+x_{i-1}, x_{i}, x_{i+1}, \ldots x_{n}\right\} \mid x\right)$. Let $G_{i}:=$ $E\left(\min \left\{k_{0}+x_{0}, k_{1}+x_{1}, \ldots k_{i-1}+x_{i-1}, x_{i}, x_{i+1}, \ldots x_{n}\right\} \mid x\right)$. By Lemma $1, F_{i}$ and $G_{i}$ are concave functions of the parameter vector $x . F_{i}$ and $G_{i}$ are also weakly increasing in $x$, by inspection.

We now proceed by induction. First $F_{0}=G_{0}$, since they are deterministically specified by $x$. Next, if $F_{i} \leq G_{i}$, then $F_{i+1} \leq G_{i+1}$. Using the laws of conditional expec- 
tation, $F_{i+1}$ can be rewritten so $F_{i+1}=E\left(E\left(\min \left\{\ell_{0}+x_{0}, \ldots, \ell_{i}+x_{i}, x_{i+1}, \ldots x_{n}\right\} \mid x, \ell_{i}\right) \mid x\right)$ Since $\ell \Uparrow k$,

$$
\begin{aligned}
E\left(E \left(\operatorname { m i n } \left\{\ell_{0}\right.\right.\right. & \left.\left.\left.+x_{0}, \ldots \ell_{i-1}+x_{i-1}, \ell_{i}+x_{i}, x_{i+1}, \ldots x_{n}\right\} \mid x, \ell_{i}\right) \mid x\right) \\
& <E\left(E\left(\min \left\{\ell_{0}+x_{0}, \ldots \ell_{i-1}+x_{i-1}, k_{i}+x_{i}, x_{i+1}, \ldots x_{n}\right\} \mid x, k_{i}\right) \mid x\right)
\end{aligned}
$$

according to Rothschild and Stiglitz [1970] because of the concavity and monotonicity of the interior conditonal expectation. By assumption, $F_{i} \leq G_{i}$, so $\forall k_{i}$,

$$
\begin{aligned}
& E\left(\min \left\{\ell_{0}+x_{0}, \ldots \ell_{i-1}+x_{i-1}, k_{i}+x_{i}, x_{i+1}, \ldots x_{n}\right\} \mid x, k_{i}\right) \\
& \quad<E\left(\min \left\{k_{0}+x_{0}, \ldots k_{i-1}+x_{i-1}, k_{i}+x_{i}, x_{i+1}, \ldots x_{n}\right\} \mid x, k_{i}\right)
\end{aligned}
$$

which implies

$$
\begin{aligned}
E\left(E \left(\operatorname { m i n } \left\{\ell_{0}\right.\right.\right. & \left.\left.\left.+x_{0}, \ldots \ell_{i-1}+x_{i-1}, k_{i}+x_{i}, x_{i+1}, \ldots x_{n}\right\} \mid x, k_{i}\right) \mid x\right) \\
& <E\left(E\left(\min \left\{k_{0}+x_{0}, \ldots k_{i-1}+x_{i-1}, k_{i}+x_{i}, x_{i+1}, \ldots x_{n}\right\} \mid x, k_{i}\right) \mid x\right)
\end{aligned}
$$

By transitivity, Eqs. (A.3) and (A.5) imply

$$
\begin{aligned}
E\left(E \left(\operatorname { m i n } \left\{\ell_{0}\right.\right.\right. & \left.\left.\left.+x_{0}, \ldots \ell_{i-1}+x_{i-1}, \ell_{i}+x_{i}, x_{i+1}, \ldots x_{n}\right\} \mid x, \ell_{i}\right) \mid x\right) \\
& <E\left(E\left(\min \left\{k_{0}+x_{0}, \ldots k_{i-1}+x_{i-1}, k_{i}+x_{i}, x_{i+1}, \ldots x_{n}\right\} \mid x, k_{i}\right) \mid x\right) .
\end{aligned}
$$

Simplifying our nested conditional expectations,

$$
\begin{aligned}
& E\left(\operatorname { m i n } \left\{\ell_{0}+x_{0}, \ldots \ell_{i-1}\right.\right.\left.\left.+x_{i-1}, \ell_{i}+x_{i}, x_{i+1}, \ldots x_{n}\right\} \mid x\right) \\
&<E\left(\min \left\{k_{0}+x_{0}, \ldots k_{i-1}+x_{i-1}, k_{i}+x_{i}, x_{i+1}, \ldots x_{n}\right\} \mid x\right)
\end{aligned}
$$

which is equivalent to $F_{i+1} \leq G_{i+1}$. Thus, by induction, $\forall n \in \mathbb{N}, F_{n} \leq G_{n}$. 
It follows directly from this that dispersal by a majorizing kernel leads to faster invasions.

\section{B Demographic stochasticity}

As in our analysis of dispersal stochasticity (Appendix A), we can represent increases in demographic stochasticity using mean-preserving spreads. Suppose we say that a progeny probability generating function $\mathcal{Q}(s)$ is related to $\mathcal{R}(s)$ by mean preserving spreads if and only if

$$
\mathcal{Q}(s)=\mathcal{R}(s)+\sum_{n=1}^{\infty} \epsilon_{n}\left[\mathcal{H}_{n}(s)-s^{n}\right]
$$

where $\mathcal{H}_{n}(s)$ is a PGF with mean $n\left(\mathcal{H}_{n}^{\prime}(1)=n\right)$, where the parameters $\epsilon_{n}$ are non-negative and sufficiently small that all the Taylor-series coefficients of $\mathcal{Q}(s)$ around $s=0$ are non-negative. Such a transform is mean-preserving [Machina and Pratt, 1997] because

$$
\mathcal{Q}^{\prime}(1)=\mathcal{R}^{\prime}(1)+\sum_{n=1}^{\infty} \epsilon_{n}\left[\mathcal{H}_{n}^{\prime}(1)-n\right]=\mathcal{R}^{\prime}(1)
$$

The variance associated with probability distributions for each $\mathcal{H}_{n}(s)$ is greater than that of a point-mass distribution with zero-variance, implying

$$
\mathcal{H}_{n}^{\prime \prime}(1) \geq n-n^{2}
$$

From this, we can show $\mathcal{Q}^{\prime \prime}(1) \geq \mathcal{R}^{\prime \prime}(1)$, and consequently that mean-preserving spreads increase variance. We can now restate Proposition 2 more precisely.

Theorem 3. If a progeny probability distribution with $P G F \mathcal{Q}(s)$ is a mean-preserving 
spread of a second progeny probability distribution with $P G F \mathcal{R}(s)$, then

$$
1-\prod_{x_{i} \in \mathcal{F}(\mathscr{N})} \mathcal{Q}\left(1-K\left(z-x_{i}\right)\right) \leq 1-\prod_{x_{i} \in \mathcal{F}(\mathscr{N})} \mathcal{R}\left(1-K\left(z-x_{i}\right)\right)
$$

Proof. By definition,

$$
\mathcal{Q}(s)=\mathcal{R}(s)+\sum_{n=1}^{\infty} \epsilon_{n}\left[\mathcal{H}_{n}(s)-s^{n}\right]
$$

By Heyde and Schuh [1978], $\mathcal{H}_{n}(s)-s^{n}$ is non-negative for $s \in[0,1]$, so $\mathcal{Q}(s) \geq \mathcal{R}(s)$ It is then a matter of direct algebraic calculation to show

$$
1-\prod_{x_{i} \in \mathcal{F}(\mathscr{N})} \mathcal{Q}\left(1-K\left(z-x_{i}\right)\right) \leq 1-\prod_{x_{i} \in \mathcal{F}(\mathscr{N})} \mathcal{R}\left(1-K\left(z-x_{i}\right)\right) .
$$

For results relating to the converse, that if $\mathcal{Q}(s) \geq \mathcal{R}(s)$ and $\mathcal{Q}^{\prime}(1)=\mathcal{R}^{\prime}(1)$, then the probability distribution of $\mathcal{Q}(s)$ can be reached by a mean-preserving spread of the probability distribution of $\mathcal{R}(s)$, see Machina and Pratt [1997].

\section{Kernel-dependent speed bounds}

Our first theorem is a lower bound on the FFD CDF. General lower bounds are difficult to construct. This is not because the math is difficult, but because they often depend on the specific details of the invasion process. Finite-state processes often have certain extinction, while contact processes and spatial branching processes have parameter-dependent thresholds. By assuming that all adults have at least 1 child, we avoid these complications and can construct a general lower bound.

Theorem 4 (Weak Lower-Bound Theorem). If $|\mathscr{N}| \geq 1$ and $\min \mathscr{N}=0$, then

$$
K(z) \leq P(z \mid \mathscr{N})
$$


Proof. To obtain the lower bound, we only need apply the approximation of Clark et al. [2001] based on the dispersals from the furthest-forward individual alone:

$$
P(z \mid \mathscr{N})=1-\prod_{x_{i} \in \mathcal{F}(\mathscr{N})} \mathcal{R}\left(1-K\left(z-x_{i}\right)\right)
$$

Since the population is non-empty and $\mathcal{R}(s) \in[0,1]$ for any probability $s$,

$$
P(z \mid \mathscr{N}) \geq 1-\mathcal{R}\left(1-K\left(z-x_{0}\right)\right)
$$

Since $\mathcal{R}(s)$ is convex, we can construct a linear upper bound.

$$
P(z \mid \mathscr{N}) \geq 1-\left[\mathcal{R}(0)+(1-\mathcal{R}(0))\left(1-K\left(z-x_{0}\right)\right)\right]
$$

and with some algebra,

$$
P(z \mid \mathscr{N}) \geq[1-\mathcal{R}(0)] K\left(z-x_{0}\right)
$$

As we have already stated in Eq. $(2.3), \mathcal{R}(0)=0$ and without loss of generality we assumed $x_{0}=0$, completing the proof.

An alternate lower bound can be constructed under the assumption that gaps between points are bounded behind the invasion front.

Theorem 5 (Strong Lower-Bound Theorem). If $|\mathscr{N}| \geq 1$, min $\mathscr{N}=0$, and there exists $\zeta \in(0, \infty)$ such that for all $x \geq 0,[x, x+\zeta] \cap \mathscr{N} \neq \emptyset$, then

$$
1-\exp \left[-\sum_{j=0}^{\infty} K(z-j \zeta)\right] \leq P(z \mid \mathscr{N})
$$

Proof. From Eq. (3.3),

$$
P(z \mid \mathscr{N})=1-\prod_{x_{i} \in \mathcal{F}(\mathscr{N})} \mathcal{R}\left(1-K\left(z-x_{i}\right)\right) .
$$


By Eq. (2.2) and (2.3), $\mathcal{R}(s)$ is convex with $\mathcal{R}(0)=r_{0}=0$ and $\mathcal{R}(1)=1$, so

$$
P(z \mid \mathscr{N}) \geq 1-\prod_{x_{i} \in \mathcal{F}(\mathscr{N})} 1-K\left(z-x_{i}\right)
$$

Since $e^{-x} \geq 1-x$, then

$$
P(z \mid \mathscr{N}) \geq 1-\exp \left[-\sum_{x_{i} \in \mathcal{F}(\mathscr{N})} K\left(z-x_{i}\right)\right]
$$

By hypothesis, the density of points must be greater than $1 / \zeta$, so that since $x_{0}=0$,

$$
\sum_{x_{i} \in \mathcal{F}(\mathscr{N})} K\left(z-x_{i}\right) \geq \sum_{j=0}^{\infty} K(z-j \zeta)
$$

This implies

$$
\exp \left[-\sum_{x_{i} \in \mathcal{F}(\mathscr{N})} K\left(z-x_{i}\right)\right] \leq \exp \left[-\sum_{j=0}^{\infty} K(z-j \zeta)\right]
$$

So we conclude

$$
P(z \mid \mathscr{N}) \geq 1-\exp \left[-\sum_{j=0}^{\infty} K(z-j \zeta)\right]
$$

To derive an upper bound on the range-expansion step-size, we make use of one of many mild generalizations of the Weierstrass product inequality [Bromwich, 1908]:

Lemma 6 (Generalized Weierstrass Product Inequality). For any finite set $W=$ $\left\{w_{0}, w_{1}, w_{2} \ldots\right\}$ where $\forall i, 0 \leq w_{i} \leq 1$,

$$
\prod_{i} \max \left\{0,1-w_{i}\right\} \geq \max \left\{0,1-\sum_{i} w_{i}\right\}
$$

Proof. We leave the proof as an excercise in induction methods for students. 
Lemma 7. For any $P G F \mathcal{R}(s)$ of a probability distribution with expected value $\mathscr{R}_{0}$, and any finite set $\left\{K_{0}, K_{1}, K_{2} \ldots\right\}$ where $\forall i, 0 \leq K_{i} \leq 1$,

$$
\prod_{i} \mathcal{R}\left(1-K_{i}\right) \geq \max \left\{0,1-\mathscr{R}_{0} \sum_{i} K_{i}\right\}
$$

Proof. First, $\mathscr{R}_{0}=\mathcal{R}^{\prime}(1)$. Since probability generating functions are convex, positive, and monotone increasing, then for any $K \in[0,1]$,

$$
\mathcal{R}(1-K) \geq \max \left\{0,1-\mathscr{R}_{0} K\right\}
$$

Extending this inequality to a product,

$$
\prod_{i} \mathcal{R}\left(1-K_{i}\right) \geq \prod_{i} \max \left\{0,1-\mathscr{R}_{0} K_{i}\right\}
$$

By the Generalized Weierstrass Product Inequality,

$$
\prod_{i} \mathcal{R}\left(1-K_{i}\right) \geq \max \left\{0,1-\mathscr{R}_{0} \sum_{i} K_{i}\right\}
$$

Using Lemma 7, we can build our upper bound on the extreme-value distribution for range expansion.

Theorem 8 (Strong Upper-Bound Theorem). Under our model assumptions,

$$
P(z \mid \mathscr{N})<\min \left\{1, \mathscr{R}_{0} \sum_{x_{i} \in \mathcal{F}(\mathscr{N})} K\left(z-x_{i}\right)\right\}
$$

Proof. Applying Lemma 7 to Eq. (3.3),

$$
P(z \mid \mathscr{N})=1-\prod_{x_{i} \in \mathcal{F}(\mathscr{N})} \mathcal{R}\left(1-K\left(z-x_{i}\right)\right) \leq 1-\max \left\{0,1-\mathscr{R}_{0} \sum_{x_{i} \in \mathcal{F}(\mathscr{N})} K\left(z-x_{i}\right)\right\}
$$


and with a little algebra,

$$
P(z \mid \mathscr{N}) \leq \min \left\{1, \mathscr{R}_{0} \sum_{x_{i} \in \mathcal{F}(\mathscr{N})} K\left(z-x_{i}\right)\right\} .
$$

So we have an upper bound on the likelihood that the leftmost individual is to the right of $z$.

The sum in our upper bound may be rewritten as

$$
P(z \mid \mathscr{N})<\min \left\{1, \mathscr{R}_{0} \int_{0}^{\infty} \mu(x) K(z-x) d x\right\}
$$

where $\mu(x)$ is a measure of the density of parent individuals. In exact calculations under a specific configuration, $\mu(x)$ must be a set of $\delta$ elta-functions corresponding to the positions of the elements of $\mathscr{N}$. But for approximation purposes, it is useful to consider a mollified measure comparable to the average shape of the wave, based on the Euler--Maclaurin summation formula or a simpler Riemann-sum inequality.

Theorem 9 (Weak Upper-Bound Theorem). Under our model hypotheses,

$$
P(z \mid \mathscr{N})<\min \left\{1, \frac{\mathscr{R}_{0}}{\gamma} \int_{0}^{\infty} K(z-x) \mathrm{d} x+\mathscr{R}_{0} K(z)\right\}
$$

Proof. From Theorem 8,

$$
P(z \mid \mathscr{N})<\min \left\{1, \mathscr{R}_{0} \sum_{x_{i} \in \mathcal{F}(\mathscr{N})} K\left(z-x_{i}\right)\right\} .
$$

Under the Lipschitz condition we have placed on our sieve and the monotonicity of $K(x)$

$$
\sum_{x_{i} \in \mathcal{F}(\mathscr{N})} K\left(z-x_{i}\right) \leq \sum_{i=0}^{\infty} K(z-i \gamma)
$$


By a Riemann sum inequality for decreasing functions,

$$
\gamma \sum_{i=1}^{\infty} K(z-i \gamma) \leq \int_{0}^{\infty} K(z-x) \mathrm{d} x
$$

SO

$$
\gamma \sum_{i=0}^{\infty} K(z-i \gamma) \leq \int_{0}^{\infty} K(z-x) \mathrm{d} x+K(z)
$$

Thus,

$$
P(z \mid \mathscr{N})<\min \left\{1, \frac{\mathscr{R}_{0}}{\gamma} \int_{0}^{\infty} K(z-x) \mathrm{d} x+\mathscr{R}_{0} K(z)\right\}
$$

The bounds on $P(z \mid \mathscr{N})$ from Theorems 4, 5, 8, and 9 are summarized by the inequalities

$$
\begin{gathered}
K(z) \leq 1-\exp \left[-\sum_{j=0}^{\infty} K(z-j \zeta)\right] \leq 1-\exp \left[-\sum_{x_{i} \in \mathcal{F}(\mathscr{N})} K\left(z-x_{i}\right)\right] \leq P(z \mid \mathscr{N}) \\
P(z \mid \mathscr{N})<\min \left\{1, \mathscr{R}_{0} \sum_{x_{i} \in \mathcal{F}(\mathscr{N})} K\left(z-x_{i}\right)\right\}<\mathscr{R}_{0}\left[K(z)+\frac{1}{\gamma} \int_{0}^{\infty} K(z-x) \mathrm{d} x\right] .
\end{gathered}
$$

where the average density of $\mathscr{N}$ on the right is between $1 / \zeta$ and $1 / \gamma$.

\section{Calculation of moments by integration}

When working with cumulative distribution functions, it is sometimes useful to express the distribution moments as integrals. Here are the most useful formulas. The expectation

$$
\langle z\rangle=\int_{-\infty}^{\infty} z P^{\prime}(z \mid X) \mathrm{d} z=f_{-\infty}^{\infty} \frac{1}{2}-P(z \mid X) \mathrm{d} z
$$


while the second moment

$$
\left\langle z^{2}\right\rangle=\int_{-\infty}^{\infty} z^{2} P^{\prime}(z \mid X) \mathrm{d} z=f_{-\infty}^{\infty}|z|-2 z P(z \mid X) \mathrm{d} z
$$

and the variance

$$
\left\langle z^{2}\right\rangle-\langle z\rangle^{2}=f_{-\infty}^{\infty}|z|-2 z P(z \mid X) \mathrm{d} z-\left(f_{-\infty}^{\infty} \frac{1}{2}-P(z \mid X) \mathrm{d} z\right)^{2}
$$

where the cross-bar is used to denote principle-value integrals. The existence of the first and second moments depends on the tail-properties of $P(z \mid X)$. In particular, if there exists $z_{0}$ such that for all $z>z_{0}, P(z \mid X)=1$, then the second moment $\left\langle z^{2}\right\rangle$ exists if and only if

$$
\lim _{z \rightarrow-\infty} z^{2} P(z \mid X)=0
$$

\section{E Calculation of effect of dispersal-delays in atom- less linear theory}

Suppose that the invasion process is deterministic, atomless, density-independent, and age-structured so

$$
b(x, t)=\int_{0}^{t} \int_{-\infty}^{\infty} A(\tau, y) b(x-y, t-\tau) d y d \tau
$$

where $b(x, t)$ is the concentration of individuals settling at location $x$ at time $t$ and the separable dispersal kernel

$$
A(t, x ; \epsilon, a, \sigma)=\mathscr{R}_{0}\left[\frac{t^{1 / \epsilon-1} e^{-t / \epsilon a}}{(\epsilon a)^{1 / \epsilon} \Gamma(1 / \epsilon)}\right]\left[\frac{e^{-\frac{x^{2}}{2 \sigma^{2}}}}{\sqrt{2 \pi \sigma^{2}}}\right]
$$


The second term in the kernel $A(t, x)$ is a Gamma distribution for dispersal delay with mean $a$ and shape parameter $\epsilon$ (so variance is $a^{2} \epsilon$ ), while the third term is a Gauss distribution for dispersal distance with mean 0 and variance $\sigma$. In the limit as $\epsilon \rightarrow 0$, all the reproduction and dispersal are concentrated exactly at time $a$, so

$$
A(t, x ; 0, a, \sigma)=\mathscr{R}_{0} \delta(t-a)\left[\frac{e^{-\frac{x^{2}}{2 \sigma^{2}}}}{\sqrt{2 \pi \sigma^{2}}}\right]
$$

We analyze travelling-wave solutions using the method of Metz et al. [2000]. The travelling wave moment generating transform is given by

$$
\mathcal{M}[A](c w, w)=\int_{0}^{\infty} \int_{-\infty}^{\infty} A(t, x) e^{-w(x+c t)} d x d t=\frac{\mathscr{R}_{0} e^{\sigma^{2} w^{2} / 2}}{(1-\epsilon a c w)^{1 / \epsilon}}
$$

and for all exponential travelling-wave solutions $\exp (-w(x+c t))$ of Eq. (E.1), $\mathcal{M}[A](c w, w)=1$. The minimum wave speed $c^{*}$ solves

$$
\frac{\partial \mathcal{M}[A]\left(c^{*} w^{*}, w^{*}\right)}{\partial w}=0
$$

If we expand our travelling wave moment generating transform in powers of $\epsilon$,

$$
\mathcal{M}[A](c w, w)=\mathscr{R}_{0} e^{-a c w+\sigma^{2} w^{2} / 2}\left(1+\epsilon a^{2} w^{2} c^{2} / 2+\mathcal{O}\left(\epsilon^{2}\right)\right)
$$

and using regular perturbation theory, we find

$$
c^{*}=\frac{\sigma \sqrt{2 \ln \left(\mathscr{R}_{0}\right)}}{a}\left[1+\epsilon \ln \left(\mathscr{R}_{0}\right)+\mathcal{O}\left(\epsilon^{2}\right)\right]
$$

Thus, if the variation in dispersal delay is small, the spread speed increases as epsilon increases $\left(\partial c^{*} / \partial \epsilon>0\right)$. Since the variance of our Gamma distribution is $a^{2} \epsilon$, this means increasing variance in our dispersal delay increases the spreading speed.

This is not particular to a Gauss spatial kernel -- we can use an arbitrary spatial 
distribution kernel (as long as $A(t, x)$ remains separable) and show that to first order,

$$
c(\epsilon)=c_{0}\left[1+\epsilon \frac{a c_{0} w_{0}}{2}+\mathcal{O}\left(\epsilon^{2}\right)\right]
$$

where $c_{0}$ and $w_{0}$ are the speed and wave number when $\epsilon=0$. Since $c_{0}$ and $w_{0}$ have the same sign, increasing the variance of our dispersal delay accelerates an invasion, at least in the limit of small $\epsilon$.

In full generality, we need to make use of the following result.

Lemma 10. A Laplace transform is always increased by a mean-preserving spread.

This is a consequence of Jensen's inequality, and is a general consequence of second-order stochastic dominance that holds for the expectation of any convex function.

Proof. Let $g(t)$ be a probability distribution over the positive reals. Then $\hat{g}(t)$ is a mean-preserving spread of $g(t)$ if and only if there exist $m(t)$ and $q(t, a)$ such that

$$
\hat{g}(t)=g(t)-\epsilon m(t)+\epsilon \int_{0}^{\infty} m(a) q(t, a) d a,
$$

where $0 \leq m(t) \leq g(t), 0 \leq \epsilon<1$, and $q(t, a)$ is a probability distribution with expectation $a$ and $q(t, a)=0$ for all $t>2 a$. Using elementary calculus, we can easily check that $\int_{0}^{\infty} t g(t) d t=\int_{0}^{\infty} t \hat{g}(t) d t$. Now, the Laplace transform of the spread distribution

$$
\mathscr{L}[\hat{g}]=\int_{0}^{\infty} \hat{g}(t) e^{-s t} d t=\mathscr{L}[g]+\epsilon \int_{0}^{\infty}\left(\int_{0}^{\infty} m(a) q(t, a) d a-m(t)\right) e^{-s t} d t
$$

Then the derivative

$$
\begin{aligned}
\frac{\partial \mathscr{L}[\hat{g}]}{\partial \epsilon} & =\int_{0}^{\infty}\left(\int_{0}^{\infty} m(a) q(t, a) d a-m(t)\right) e^{-s t} d t \\
& =\int_{0}^{\infty} m(a) \int_{0}^{\infty} q(t, a) e^{-s t} d t d a-\int_{0}^{\infty} m(t) e^{-s t} d t
\end{aligned}
$$


Since the exponential function is convex and $\int_{0}^{\infty} t q(t, a) d t=a$, then by Jensen's inequality, $\int_{0}^{\infty} q(t, a) e^{-s t} d t \geq e^{-s a}$. It follows by substitution that $\frac{\partial \mathscr{L}[\hat{g}]}{\partial \epsilon} \geq 0$. Since we have applied here an arbitrary mean-preserving spread, we can conclude that all mean-preserving spreads increase the Laplace transform's value.

Theorem 11. The minimum spreading speed of atomless deterministic linear system (E.1) with seperable kernel increases as stochasticity in dispersal timing is increased but the mean dispersal time is constant.

Proof. Let $\hat{A}(t, x ; \epsilon)$ be a separable kernel for (E.1) with mean-spread parameter $\epsilon$ as above, so we can write $\hat{A}(t, x ; \epsilon)=\hat{g}(t ; \epsilon) k(x)$. Then $F(w)$ be the moment generating transform of $k(x)$, while $L(s ; \epsilon)$ is the Laplace transform of $\hat{g}(t ; \epsilon)$. The traveling wave moment generating transform of $\hat{A}(t, x ; \epsilon)$ can then be calculated, so

$$
\mathcal{M}[\hat{A} ; \epsilon](s, w)=\int_{-\infty}^{\infty} \int_{0}^{\infty} \hat{g}(t ; \epsilon) k(x) e^{-s t-w x} d t d x=L(s ; \epsilon) F(w)
$$

The moment generating function $\mathcal{M}[\hat{A} ; \epsilon](s, w)$ is positive, convex in $s$ and $w$, and decreasing in $s$. By Lemma $10, \partial \mathcal{M} / \partial \epsilon \geq 0$. Since for all travelling wave solutions, $\mathcal{M}[\hat{A} ; \epsilon](c w, w)=1$, we can deduce with some geometric reasoning that $\partial c(w ; \epsilon) / \partial \epsilon \geq$ 0. Since the mean spread increases the spread at all wave numbers, it must increase the spread at the minimizing wave number $\left(\partial c^{*} / \partial \epsilon \geq 0\right)$. 